\title{
NUCLÉAIRE ET ENVIRONNEMENT
}

$N_{u}$ ne prétendons pas que les articles publiés ici l'épuisent en entier, aussi prestigieuse que soit la notoriété de leurs auteurs. Le dossier présenté fournit néanmoins de très solides points de repères dans les domaines essentiels qui sont à la base même du sujet : l'étude de l'écosphère, la radioactivité, l'impact des installations nucléaires en fonctionnement normal et en situation accidentelle, l'évaluation et la prise en compte des séismes... Loin des débats trop passionnés et de l'atmosphère de croisade qui souvent prévaut lorsque ces thèmes viennent en discussion dans l'espace public, les textes publiés constituent des développements objectifs, fondés sur des données mesurables, faisant la part des incertitudes lorsqu'elles existent et n'ayant d'autre cause à défendre que celle de la rigueur scientifique dans l'acquisition et l'exposé des connaissances. Nous remercions les auteurs pour leurs excellentes contributions ainsi que Jacques Poirier pour la part qu'il a prise dans la conception et la réalisation de ce dossier.

Francis SORIN 